CRYSTALLOGRAPHIC COMMUNICATIONS

ISSN 2056-9890

Received 18 March 2017

Accepted 28 March 2017

Edited by P. C. Healy, Griffith University, Australia

Keywords: crystal structure; hydrogen bonds; piperidine; sulfate; dimethylthiourea.

CCDC reference: 1540583

Supporting information: this article has supporting information at journals.iucr.org/e

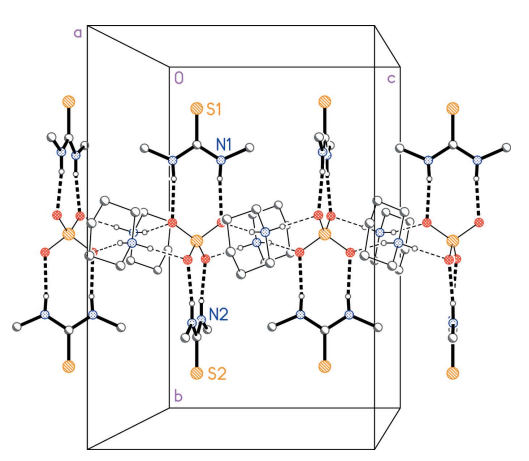

OPEN $\odot$ ACCESS

\section{Crystal structure of the 1:2 adduct of bis(piperidinium) sulfate and 1,3-dimethylthiourea}

\author{
Cindy Döring, Julian F. D. Lueck and Peter G. Jones*
}

Institut für Anorganische und Analytische Chemie, Technische Universität Braunschweig, Postfach 3329, D-38023 Braunschweig, Germany. *Correspondence e-mail: p.jones@tu-bs.de

In the title compound, $2 \mathrm{C}_{5} \mathrm{H}_{12} \mathrm{~N}^{+} \cdot \mathrm{SO}_{4}{ }^{2-} \cdot 2 \mathrm{C}_{3} \mathrm{H}_{8} \mathrm{~N}_{2} \mathrm{~S}$, the $\mathrm{C}=\mathrm{S}$ groups of the two independent 1,3-dimethylurea molecules and the sulfur atom of the anion lie on twofold axes. The packing is centred on bis(piperidinium) sulfate ribbons parallel to the $c$ axis; the cations are hydrogen bonded to the sulfate by $\mathrm{N}-$ $\mathrm{H} \cdots \mathrm{O}$ and $\mathrm{C}-\mathrm{H} \cdots \mathrm{O}$ interactions. The 1,3-dimethylurea molecules are also hydrogen bonded to sulfate $\mathrm{O}$ atoms, and project outwards from the ribbon parallel to the $b$ axis.

\section{Chemical context}

We are interested in the structures of adducts of urea and thiourea, and simple derivatives of these compounds, with neutral molecules. We have published two reports on adducts of dioxane and morpholine with various methylthioureas (Jones et al., 2013; Taouss \& Jones, 2016). In the course of our current investigations, we attempted to obtain adducts of methylthioureas with piperidine, although monoamines are not good adduct partners for ureas and thioureas. Indeed, no simple adducts were obtained. In one case, however, we overlayered a solution of 1,3-dimethylthiourea (1,3-DMT) in piperidine with diethyl ether and obtained colourless crystals, the structure of which is reported here.

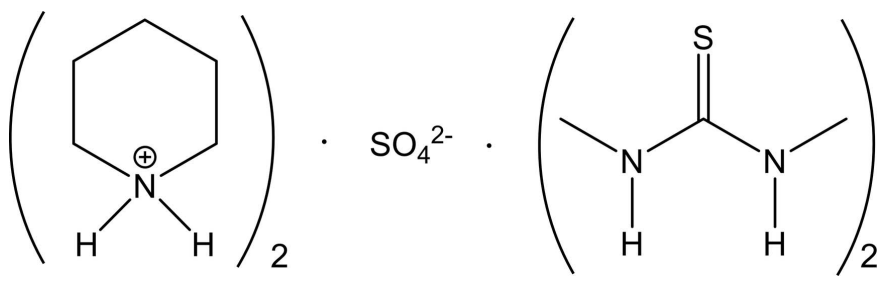

\section{Structural commentary}

The crystals proved to be a 1:2 adduct of bis(piperidinium) sulfate and 1,3-DMT (Fig. 1), with the sulfate anion presumably generated by partial hydrolysis and/or decomposition of the 1,3-DMT under the influence of peroxides in the ether. The $\mathrm{C}=\mathrm{S}$ bonds of both 1,3-DMT molecules lie along twofold axes. The sulfate sulfur atom also lies on a twofold axis. The piperidine lies on a general position. Molecular dimensions may be regarded as normal. Both 1,3-DMT molecules are essentially planar (r.m.s. deviation of non-H atoms: 0.004 and $0.010 \AA$ ). Both NH functions of each 1,3-DMT are trans to the $\mathrm{C}=\mathrm{S}$ double bond across the respective $\mathrm{C}-\mathrm{N}$ bond (associated with the hydrogen-bonding pattern, see below), so that 
Table 1

Hydrogen-bond geometry $\left(\AA,^{\circ}\right)$.

\begin{tabular}{lllll}
\hline$D-\mathrm{H} \cdots A$ & $D-\mathrm{H}$ & $\mathrm{H} \cdots A$ & $D \cdots A$ & $D-\mathrm{H} \cdots A$ \\
\hline $\mathrm{N} 1-\mathrm{H} 03 \cdots \mathrm{O} 1$ & $0.86(3)$ & $2.06(3)$ & $2.9062(18)$ & $173(2)$ \\
$\mathrm{N} 2-\mathrm{H} 04 \cdots \mathrm{O} 2$ & $0.89(2)$ & $2.00(2)$ & $2.8874(17)$ & $172(2)$ \\
$\mathrm{N} 11-\mathrm{H} 01 \cdots \mathrm{O} 1^{\mathrm{i}}$ & $0.83(2)$ & $1.98(2)$ & $2.7953(18)$ & $167.4(19)$ \\
$\mathrm{N} 11-\mathrm{H} 02 \cdots \mathrm{O} 2$ & $0.91(2)$ & $1.85(2)$ & $2.7589(17)$ & $176(2)$ \\
$\mathrm{C} 12-\mathrm{H} 12 A \cdots \mathrm{S} 1^{\mathrm{ii}}$ & 0.99 & 2.94 & $3.8213(17)$ & 150 \\
$\mathrm{C} 15-\mathrm{H} 15 A \cdots \mathrm{O} 2^{\mathrm{iii}}$ & 0.99 & 2.49 & $3.435(2)$ & 158 \\
\hline
\end{tabular}

Symmetry codes: (i) $-x+1,-y+1,-z+1$; (ii) $x-\frac{1}{2}, y+\frac{1}{2}, z$; (iii) $x,-y+1, z+\frac{1}{2}$

the methyl groups are cis, with $\mathrm{C}_{\text {methyl }}-\mathrm{N}-\mathrm{C}=\mathrm{S}$ torsion angles are close to zero $\left[\mathrm{C} 11-\mathrm{N} 1-\mathrm{C} 1-\mathrm{S} 1=0.9(2)^{\circ}\right.$ and $\left.\mathrm{S} 2-\mathrm{C} 2-\mathrm{N} 2-\mathrm{C} 21=-2.05(17)^{\circ}\right]$. Free $1,3-\mathrm{DMT}$ crystallizes with four independent molecules, each of which has one $\mathrm{NH}$ group cis and one trans to $\mathrm{C}=\mathrm{S}$, but the structure is severely disordered (Jones et al., 2013).

\section{Supramolecular features}

The packing is based on bis(piperidinium) sulfate ribbons parallel to the $c$ axis in the region $x, y \simeq 1 / 2$ (Fig. 2) and also at $x, y \simeq 0$, etc.; the cations are hydrogen bonded to the sulfate by $\mathrm{N}-\mathrm{H} \cdots \mathrm{O}$ interactions, as expected, but also by a short interaction $\mathrm{C} 15-\mathrm{H} 15 A \cdots \mathrm{O} 2$ (Table 1 ). Each pair of successive sulfate ions in the ribbon is bridged by two piperidinium cations. The 1,3-DMT molecules are also hydrogen bonded to sulfate oxygens (Figs. 1 and 3); each 1,3-DMT bridges two oxygens of the same anion and projects outwards from the ribbons parallel to the $b$ axis. In the presence of the sulfate oxygen atoms as strong hydrogen-bond acceptors, the 1,3DMT sulfur atoms do not accept any classical hydrogen bonds.

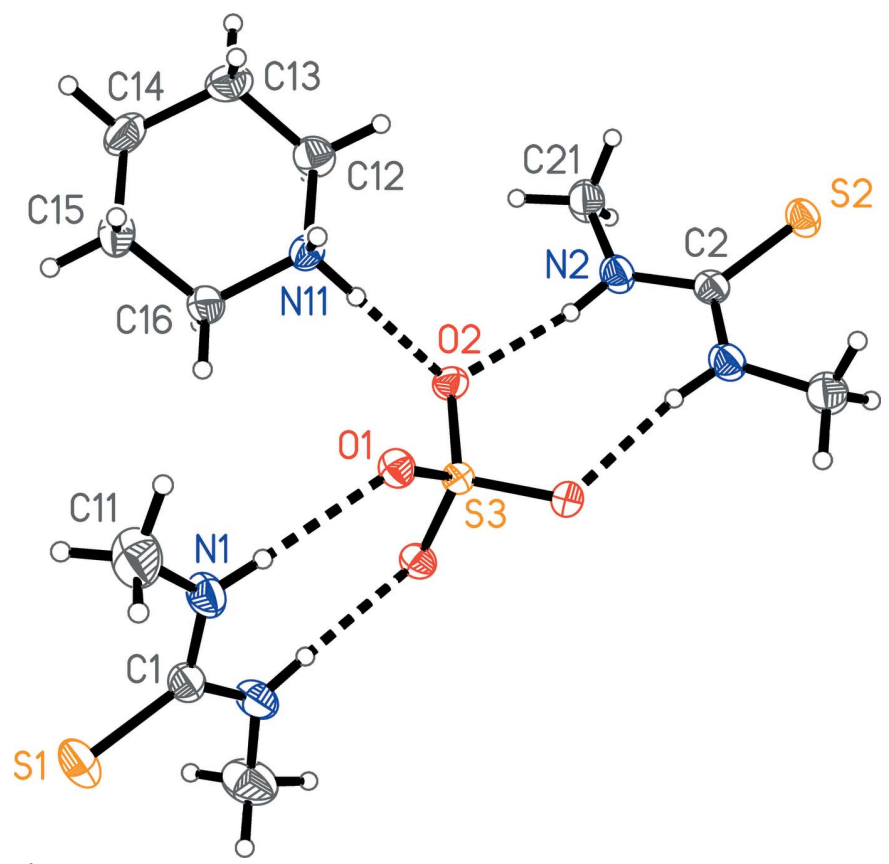

Figure 1

The structure of the title compound in the crystal. Only the asymmetric unit is labelled. Displacement ellipsoids represent 50\% probability levels. The dashed lines represent hydrogen bonds.

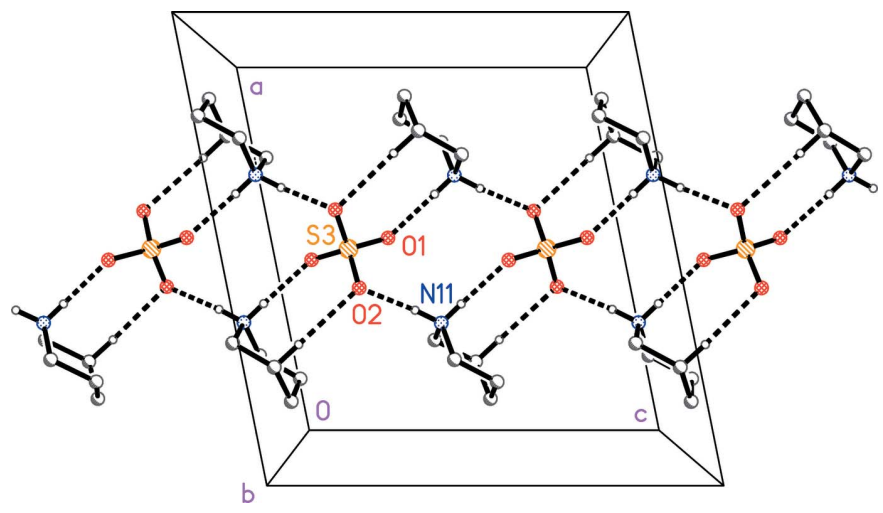

Figure 2

Packing diagram of the title compound: the bis(piperidinium) sulfate substructure viewed parallel to the $b$ axis. Dashed lines represent hydrogen bonds. Hydrogen atoms not involved in hydrogen bonds are omitted for clarity.

\section{Database survey}

A search of the Cambridge Database (Version 1.19; Groom et al., 2016) found three adducts of 1,3-DMT, excluding metal complexes. The 1:2 adduct between 18-crown-6 and 1,3-DMT (Weber, 1983) also displays a trans geometry for both $\mathrm{NH}$ functions, but the 1:2 adduct between 1,4-dioxane and 1,3DMT (Jones et al., 2013) and a 1,3-DMT adduct of a 1,3-DMTgold(I) complex (Eikens et al., 1994) both have one NH function cis and one trans. Only one other piperidinium sulfate derivative was found, namely tris(piperidinium) hydrogensulfate sulfate (Lukianova et al., 2015).

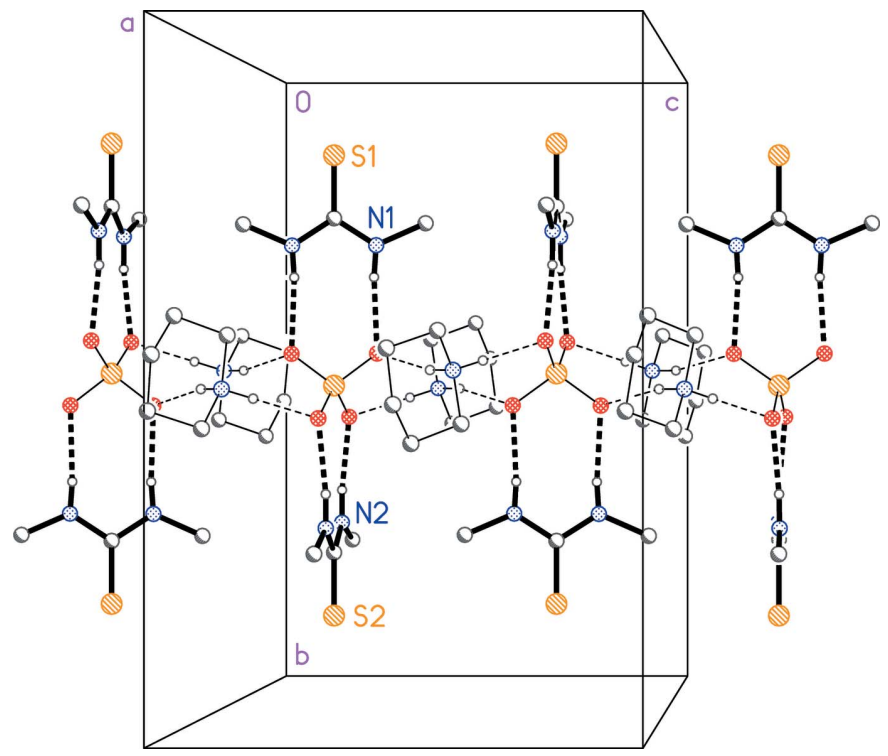

Figure 3

Packing diagram of the title compound: attachment of the thiourea molecules to the bis(piperidinium) sulfate chain, viewed perpendicular to the $b c$ plane. Dashed lines represent hydrogen bonds. Methylene hydrogen atoms are omitted for clarity. 
Table 2

Experimental details.

\begin{tabular}{|c|c|}
\hline \multicolumn{2}{|l|}{ Crystal data } \\
\hline Chemical formula & $2 \mathrm{C}_{5} \mathrm{H}_{12} \mathrm{~N}^{+} \cdot \mathrm{SO}_{4}{ }^{2-} \cdot 2 \mathrm{C}_{3} \mathrm{H}_{8} \mathrm{~N}_{2} \mathrm{~S}$ \\
\hline$M_{\mathrm{r}}$ & 476.72 \\
\hline Crystal system, space group & Monoclinic, $C 2 / c$ \\
\hline Temperature $(\mathrm{K})$ & 100 \\
\hline$a, b, c(\AA)$ & $\begin{array}{l}12.5899(5), 17.5691(6), \\
11.8980(5)\end{array}$ \\
\hline$\beta\left({ }^{\circ}\right)$ & $101.326(4)$ \\
\hline$V\left(\AA^{3}\right)$ & $2580.52(17)$ \\
\hline$Z$ & 4 \\
\hline Radiation type & $\mathrm{Cu} \mathrm{K \alpha}$ \\
\hline$\mu\left(\mathrm{mm}^{-1}\right)$ & 2.89 \\
\hline Crystal size $(\mathrm{mm})$ & $0.25 \times 0.05 \times 0.02$ \\
\hline \multicolumn{2}{|l|}{ Data collection } \\
\hline Diffractometer & $\begin{array}{l}\text { Oxford Diffraction Xcalibur, Atlas, } \\
\text { Nova }\end{array}$ \\
\hline Absorption correction & $\begin{array}{l}\text { Multi-scan (CrysAlis PRO; } \\
\quad \text { Agilent, 2014) }\end{array}$ \\
\hline$T_{\min }, T_{\max }$ & $0.514,1.000$ \\
\hline $\begin{array}{l}\text { No. of measured, independent and } \\
\text { observed }[I>2 \sigma(I)] \text { reflections }\end{array}$ & $21112,2701,2295$ \\
\hline$R_{\text {int }}$ & 0.073 \\
\hline$(\sin \theta / \lambda)_{\max }\left(\AA^{-1}\right)$ & 0.630 \\
\hline \multicolumn{2}{|l|}{ Refinement } \\
\hline$R\left[F^{2}>2 \sigma\left(F^{2}\right)\right], w R\left(F^{2}\right), S$ & $0.034,0.084,1.03$ \\
\hline No. of reflections & 2701 \\
\hline No. of parameters & 152 \\
\hline $\mathrm{H}$-atom treatment & $\begin{array}{l}\mathrm{H} \text { atoms treated by a mixture of } \\
\text { independent and constrained } \\
\text { refinement }\end{array}$ \\
\hline$\Delta \rho_{\max }, \Delta \rho_{\min }\left(\mathrm{e} \AA^{-3}\right)$ & $0.21,-0.38$ \\
\hline
\end{tabular}

Computer programs: CrysAlis PRO (Agilent, 2014), SHELXS97, SHELXL97 and XP (Sheldrick, 2008).

\section{Synthesis and crystallization}

$208 \mathrm{mg}$ (2 mmol) 1,3-DMT were dissolved in $2 \mathrm{~mL}$ piperidine. The solution was overlayered with diethyl ether. Colourless needles formed overnight.

\section{Refinement}

Crystal data, data collection and structure refinement details are summarized in Table 2. The asymmetric unit was chosen such that the occupied twofold axis is $\frac{1}{2}, y, \frac{1}{4}$. The $\mathrm{NH}$ hydrogen atoms were refined freely. The $\mathrm{H}$ atoms of the methyl groups were identified in a difference synthesis, idealized and refined as rigid groups allowed to rotate but not tip $(\mathrm{C}-\mathrm{H} 0.98 \AA$, $\left.\mathrm{H}-\mathrm{C}-\mathrm{H} 109.5^{\circ}\right)$. Methylene $\mathrm{H}$ atoms were included using a riding model starting from calculated positions ( $\mathrm{C}-\mathrm{H} 0.99 \AA$ А $)$.

\section{References}

Agilent (2014). CrysAlis PRO. Agilent Technologies Ltd, Yarnton, England.

Eikens, W., Jones, P. G., Lautner, J. \& Thöne, C. (1994). Z. Naturforsch. Teil B, 49, 21-26.

Groom, C. R., Bruno, I. J., Lightfoot, M. P. \& Ward, S. C. (2016). Acta Cryst. B72, 171-179.

Jones, P. G., Taouss, C., Teschmit, N. \& Thomas, L. (2013). Acta Cryst. B69, 405-413.

Lukianova, T. J., Kinzhybalo, V. \& Pietraszko, A. (2015). Acta Cryst. E71, 1444-1446.

Sheldrick, G. M. (2008). Acta Cryst. A64, 112-122.

Taouss, C. \& Jones, P. G. (2016). Z. Naturforsch. Teil B, 71, 905-907.

Weber, G. (1983). Acta Cryst. C39, 896-899. 


\section{supporting information}

Acta Cryst. (2017). E73, 651-653 [https://doi.org/10.1107/S2056989017004820]

\section{Crystal structure of the 1:2 adduct of bis(piperidinium) sulfate and 1,3-di- methylthiourea}

\section{Cindy Döring, Julian F. D. Lueck and Peter G. Jones}

Computing details

Data collection: CrysAlis PRO (Agilent, 2014); cell refinement: CrysAlis PRO (Agilent, 2014); data reduction: CrysAlis PRO (Agilent, 2014); program(s) used to solve structure: SHELXS97 (Sheldrick, 2008); program(s) used to refine structure: SHELXL97 (Sheldrick, 2008); molecular graphics: XP (Sheldrick, 2008); software used to prepare material for publication: SHELXL97 (Sheldrick, 2008).

Bis(piperidin-1-ium) sulfate-1,3-dimethylthiourea (1/2)

Crystal data

$2 \mathrm{C}_{5} \mathrm{H}_{12} \mathrm{~N}^{+} \cdot \mathrm{SO}_{4}{ }^{2-} \cdot 2 \mathrm{C}_{3} \mathrm{H}_{8} \mathrm{~N}_{2} \mathrm{~S}$

$M_{r}=476.72$

Monoclinic, $C 2 / c$

Hall symbol: $-\mathrm{C} 2 \mathrm{yc}$

$a=12.5899(5) \AA$

$b=17.5691(6) \AA$

$c=11.8980(5) \AA$

$\beta=101.326(4)^{\circ}$

$V=2580.52(17) \AA^{3}$

$Z=4$

\section{Data collection}

Oxford Diffraction Xcalibur, Atlas, Nova diffractometer

Radiation source: sealed X-ray tube

Mirror monochromator

Detector resolution: 10.3543 pixels $\mathrm{mm}^{-1}$

$\omega$ scan

Absorption correction: multi-scan

(CrysAlisPro; Agilent, 2014)

$T_{\min }=0.514, T_{\max }=1.000$

\section{Refinement}

Refinement on $F^{2}$

Least-squares matrix: full

$R\left[F^{2}>2 \sigma\left(F^{2}\right)\right]=0.034$

$w R\left(F^{2}\right)=0.084$

$S=1.03$

2701 reflections

152 parameters

0 restraints
$F(000)=1032$

$D_{\mathrm{x}}=1.227 \mathrm{Mg} \mathrm{m}^{-3}$

$\mathrm{Cu} K \alpha$ radiation, $\lambda=1.54184 \AA$

Cell parameters from 6031 reflections

$\theta=4.4-76.2^{\circ}$

$\mu=2.89 \mathrm{~mm}^{-1}$

$T=100 \mathrm{~K}$

Lath, colourless

$0.25 \times 0.05 \times 0.02 \mathrm{~mm}$

21112 measured reflections

2701 independent reflections

2295 reflections with $I>2 \sigma(I)$

$R_{\text {int }}=0.073$

$\theta_{\max }=76.2^{\circ}, \theta_{\min }=4.4^{\circ}$

$h=-15 \rightarrow 15$

$k=-22 \rightarrow 22$

$l=-14 \rightarrow 14$

Primary atom site location: structure-invariant direct methods

Secondary atom site location: difference Fourier map

Hydrogen site location: inferred from neighbouring sites

$\mathrm{H}$ atoms treated by a mixture of independent and constrained refinement 
$w=1 /\left[\sigma^{2}\left(F_{\mathrm{o}}^{2}\right)+(0.0415 P)^{2}+0.6886 P\right]$

where $P=\left(F_{\mathrm{o}}^{2}+2 F_{\mathrm{c}}{ }^{2}\right) / 3$

$(\Delta / \sigma)_{\max }=0.001$

$$
\begin{aligned}
\Delta \rho_{\max } & =0.21 \mathrm{e} \AA^{-3} \\
\Delta \rho=-0.38 & \AA^{-3}
\end{aligned}
$$

$\Delta \rho_{\min }=-0.38$ e $\AA^{-3}$

\section{Special details}

Geometry. All esds (except the esd in the dihedral angle between two 1.s. planes) are estimated using the full covariance matrix. The cell esds are taken into account individually in the estimation of esds in distances, angles and torsion angles; correlations between esds in cell parameters are only used when they are defined by crystal symmetry. An approximate (isotropic) treatment of cell esds is used for estimating esds involving 1.s. planes.

Refinement. Refinement of $\mathrm{F}^{2}$ against ALL reflections. The weighted $\mathrm{R}$-factor $\mathrm{wR}$ and goodness of fit $\mathrm{S}$ are based on $\mathrm{F}^{2}$, conventional R-factors $R$ are based on $F$, with $F$ set to zero for negative $F^{2}$. The threshold expression of $F^{2}>2$ sigma $\left(F^{2}\right)$ is used only for calculating R-factors(gt) etc. and is not relevant to the choice of reflections for refinement. R-factors based on $\mathrm{F}^{2}$ are statistically about twice as large as those based on F, and R-factors based on ALL data will be even larger.

Fractional atomic coordinates and isotropic or equivalent isotropic displacement parameters $\left(\AA^{2}\right)$

\begin{tabular}{|c|c|c|c|c|}
\hline & $x$ & $y$ & $z$ & $U_{\text {iso }} * / U_{\text {eq }}$ \\
\hline S1 & 0.5000 & $0.15866(3)$ & 0.2500 & $0.03441(16)$ \\
\hline N1 & $0.50629(13)$ & $0.29584(8)$ & $0.34615(12)$ & 0.0309 (3) \\
\hline H03 & $0.509(2)$ & $0.3444(14)$ & $0.342(2)$ & $0.043(6)^{*}$ \\
\hline $\mathrm{C} 1$ & 0.5000 & $0.25465(12)$ & 0.2500 & $0.0257(4)$ \\
\hline C11 & $0.5151(2)$ & $0.26298(12)$ & $0.45902(17)$ & $0.0474(5)$ \\
\hline H11A & 0.4555 & 0.2271 & 0.4586 & $0.071 *$ \\
\hline H11B & 0.5116 & 0.3035 & 0.5148 & $0.071 *$ \\
\hline $\mathrm{H} 11 \mathrm{C}$ & 0.5844 & 0.2361 & 0.4802 & $0.071^{*}$ \\
\hline $\mathrm{S} 2$ & 0.5000 & $0.85910(3)$ & 0.2500 & $0.02659(14)$ \\
\hline $\mathrm{C} 2$ & 0.5000 & $0.76309(12)$ & 0.2500 & $0.0225(4)$ \\
\hline N11 & $0.32010(11)$ & $0.51380(7)$ & $0.44604(12)$ & 0.0221 \\
\hline H01 & $0.3664(18)$ & $0.5141(11)$ & 0.5057 (19) & $0.024(5)^{*}$ \\
\hline H02 & $0.3518(18)$ & $0.5281(12)$ & $0.387(2)$ & $0.033(5)^{*}$ \\
\hline $\mathrm{C} 12$ & $0.23562(13)$ & $0.57046(9)$ & $0.46151(15)$ & 0.0298 \\
\hline $\mathrm{H} 12 \mathrm{~A}$ & 0.1816 & 0.5756 & 0.3892 & $0.036^{*}$ \\
\hline H12B & 0.2697 & 0.6208 & 0.4809 & $0.036^{*}$ \\
\hline C13 & $0.18007(15)$ & $0.54456(11)$ & $0.55658(17)$ & $0.0361(4)$ \\
\hline H13A & 0.1217 & 0.5809 & 0.5640 & $0.043^{*}$ \\
\hline H13B & 0.2330 & 0.5441 & 0.6301 & $0.043^{*}$ \\
\hline $\mathrm{C} 14$ & $0.13203(15)$ & $0.46502(11)$ & $0.53210(16)$ & $0.0347(4)$ \\
\hline $\mathrm{H} 14 \mathrm{~A}$ & 0.0996 & 0.4481 & 0.5972 & $0.042 *$ \\
\hline H14B & 0.0739 & 0.4666 & 0.4627 & $0.042 *$ \\
\hline C15 & $0.21891(14)$ & $0.40868(10)$ & $0.51400(14)$ & $0.0305(4)$ \\
\hline H15A & 0.2731 & 0.4031 & 0.5860 & $0.037^{*}$ \\
\hline H15B & 0.1855 & 0.3583 & 0.4936 & $0.037^{*}$ \\
\hline C16 & $0.27441(13)$ & $0.43579(9)$ & $0.41936(15)$ & $0.0274(3)$ \\
\hline H16A & 0.3334 & 0.4001 & 0.4114 & $0.033^{*}$ \\
\hline H16B & 0.2217 & 0.4367 & 0.3457 & $0.033 *$ \\
\hline S3 & 0.5000 & 0.50921 & 0.2500 & $0.01895(12)$ \\
\hline $\mathrm{O} 1$ & $0.52691(9)$ & $0.46061(6)$ & $0.35314(9)$ & $0.0235(2)$ \\
\hline $\mathrm{O} 2$ & $0.40655(9)$ & $0.55785(6)$ & $0.26031(9)$ & $0.0233(2)$ \\
\hline N2 & $0.40879(11)$ & $0.72181(8)$ & $0.24440(12)$ & $0.0267(3)$ \\
\hline
\end{tabular}




$\begin{array}{lllll}\text { H04 } & 0.4138(19) & 0.6711(13) & 0.246(2) & 0.036(6)^{*} \\ \text { C21 } & 0.30262(14) & 0.75398(10) & 0.24149(17) & 0.0331(4) \\ \text { H21A } & 0.2771 & 0.7787 & 0.1673 & 0.050^{*} \\ \text { H21B } & 0.3067 & 0.7916 & 0.3030 & 0.050^{*} \\ \text { H21C } & 0.2521 & 0.7134 & 0.2522 & 0.050^{*}\end{array}$

Atomic displacement parameters $\left(\AA^{2}\right)$

\begin{tabular}{lllllll}
\hline & $U^{11}$ & $U^{22}$ & $U^{33}$ & $U^{12}$ & $U^{13}$ & $U^{23}$ \\
\hline S1 & $0.0329(3)$ & $0.0197(3)$ & $0.0475(4)$ & 0.000 & $0.0000(3)$ & 0.000 \\
N1 & $0.0440(8)$ & $0.0225(7)$ & $0.0268(7)$ & $-0.0030(6)$ & $0.0080(6)$ & $0.0015(5)$ \\
C1 & $0.0233(10)$ & $0.0230(10)$ & $0.0304(12)$ & 0.000 & $0.0047(9)$ & 0.000 \\
C11 & $0.0703(15)$ & $0.0421(11)$ & $0.0295(10)$ & $-0.0070(10)$ & $0.0092(10)$ & $0.0057(8)$ \\
S2 & $0.0272(3)$ & $0.0187(2)$ & $0.0334(3)$ & 0.000 & $0.0047(2)$ & 0.000 \\
C2 & $0.0274(11)$ & $0.0228(10)$ & $0.0170(9)$ & 0.000 & $0.0038(8)$ & 0.000 \\
N11 & $0.0200(6)$ & $0.0247(6)$ & $0.0219(6)$ & $-0.0015(5)$ & $0.0046(6)$ & $-0.0001(5)$ \\
C12 & $0.0247(8)$ & $0.0268(8)$ & $0.0375(9)$ & $0.0050(6)$ & $0.0047(7)$ & $0.0004(6)$ \\
C13 & $0.0282(8)$ & $0.0425(10)$ & $0.0408(10)$ & $-0.0003(7)$ & $0.0145(8)$ & $-0.0107(8)$ \\
C14 & $0.0262(8)$ & $0.0466(10)$ & $0.0347(9)$ & $-0.0073(7)$ & $0.0141(7)$ & $-0.0030(7)$ \\
C15 & $0.0303(8)$ & $0.0301(8)$ & $0.0304(8)$ & $-0.0075(6)$ & $0.0041(7)$ & $0.0025(6)$ \\
C16 & $0.0250(8)$ & $0.0258(8)$ & $0.0323(9)$ & $-0.0036(6)$ & $0.0077(6)$ & $-0.0051(6)$ \\
S3 & $0.0194(2)$ & $0.0182(2)$ & $0.0195(2)$ & 0.000 & $0.00437(18)$ & 0.000 \\
O1 & $0.0262(5)$ & $0.0221(5)$ & $0.0217(5)$ & $-0.0004(4)$ & $0.0033(4)$ & $0.0014(4)$ \\
O2 & $0.0224(5)$ & $0.0219(5)$ & $0.0270(6)$ & $0.0033(4)$ & $0.0085(4)$ & $0.0017(4)$ \\
N2 & $0.0289(7)$ & $0.0200(6)$ & $0.0310(7)$ & $-0.0007(5)$ & $0.0056(6)$ & $0.0008(5)$ \\
C21 & $0.0284(8)$ & $0.0282(8)$ & $0.0428(10)$ & $-0.0022(6)$ & $0.0071(7)$ & $0.0009(7)$ \\
& & & & & &
\end{tabular}

Geometric parameters $\left(\AA,{ }^{\circ}\right)$

\begin{tabular}{llll}
\hline $\mathrm{S} 1-\mathrm{C} 1$ & $1.687(2)$ & $\mathrm{C} 13-\mathrm{H} 13 \mathrm{~A}$ & 0.9900 \\
$\mathrm{~N} 1-\mathrm{C} 1$ & $1.3428(19)$ & $\mathrm{C} 13-\mathrm{H} 13 \mathrm{~B}$ & 0.9900 \\
$\mathrm{~N} 1-\mathrm{C} 11$ & $1.446(2)$ & $\mathrm{C} 14-\mathrm{C} 15$ & $1.522(3)$ \\
$\mathrm{N} 1-\mathrm{H} 03$ & $0.86(3)$ & $\mathrm{C} 14-\mathrm{H} 14 \mathrm{~A}$ & 0.9900 \\
$\mathrm{C} 1-\mathrm{N} 1 \mathrm{i}$ & $1.3427(19)$ & $\mathrm{C} 14-\mathrm{H} 14 \mathrm{~B}$ & 0.9900 \\
$\mathrm{C} 11-\mathrm{H} 11 \mathrm{~A}$ & 0.9800 & $\mathrm{C} 15-\mathrm{C} 16$ & $1.514(2)$ \\
$\mathrm{C} 11-\mathrm{H} 11 \mathrm{~B}$ & 0.9800 & $\mathrm{C} 15-\mathrm{H} 15 \mathrm{~A}$ & 0.9900 \\
$\mathrm{C} 11-\mathrm{H} 11 \mathrm{C}$ & 0.9800 & $\mathrm{C} 15-\mathrm{H} 15 \mathrm{~B}$ & 0.9900 \\
$\mathrm{~S} 2-\mathrm{C} 2$ & $1.687(2)$ & $\mathrm{C} 16-\mathrm{H} 16 \mathrm{~A}$ & 0.9900 \\
$\mathrm{C} 2-\mathrm{N} 2$ & $1.3487(18)$ & $\mathrm{C} 16-\mathrm{H} 16 \mathrm{~B}$ & 0.9900 \\
$\mathrm{C} 2-\mathrm{N} 2 \mathrm{i}$ & $1.3488(18)$ & $\mathrm{S} 3-\mathrm{O} 2$ & $1.4786(10)$ \\
$\mathrm{N} 11-\mathrm{C} 12$ & $1.494(2)$ & $\mathrm{S} 3-\mathrm{O} 2^{\mathrm{i}}$ & $1.4786(10)$ \\
$\mathrm{N} 11-\mathrm{C} 16$ & $1.4961(19)$ & $\mathrm{S} 3-\mathrm{O} 1^{\mathrm{i}}$ & $1.4787(11)$ \\
$\mathrm{N} 11-\mathrm{H} 01$ & $0.83(2)$ & $\mathrm{S} 3-\mathrm{O} 1$ & $1.4787(11)$ \\
$\mathrm{N} 11-\mathrm{H} 02$ & $0.91(2)$ & $\mathrm{N} 2-\mathrm{C} 21$ & $1.445(2)$ \\
$\mathrm{C} 12-\mathrm{C} 13$ & $1.512(3)$ & $\mathrm{N} 2-\mathrm{H} 04$ & $0.89(2)$ \\
$\mathrm{C} 12-\mathrm{H} 12 \mathrm{~A}$ & 0.9900 & $\mathrm{C} 21-\mathrm{H} 21 \mathrm{~A}$ & 0.9800 \\
$\mathrm{C} 12-\mathrm{H} 12 \mathrm{~B}$ & 0.9900 & $\mathrm{C} 21-\mathrm{H} 21 \mathrm{~B}$ & 0.9800 \\
$\mathrm{C} 13-\mathrm{C} 14$ & $1.528(3)$ & $\mathrm{C} 21-\mathrm{H} 21 \mathrm{C}$ & 0.9800
\end{tabular}




\begin{tabular}{|c|c|c|c|}
\hline $\mathrm{C} 1-\mathrm{N} 1-\mathrm{C} 11$ & $123.85(16)$ & $\mathrm{C} 15-\mathrm{C} 14-\mathrm{C} 13$ & $110.69(14)$ \\
\hline $\mathrm{C} 1-\mathrm{N} 1-\mathrm{H} 03$ & $119.0(16)$ & $\mathrm{C} 15-\mathrm{C} 14-\mathrm{H} 14 \mathrm{~A}$ & 109.5 \\
\hline $\mathrm{C} 11-\mathrm{N} 1-\mathrm{H} 03$ & $117.0(16)$ & $\mathrm{C} 13-\mathrm{C} 14-\mathrm{H} 14 \mathrm{~A}$ & 109.5 \\
\hline $\mathrm{N} 1{ }^{\mathrm{i}}-\mathrm{C} 1-\mathrm{N} 1$ & $114.8(2)$ & $\mathrm{C} 15-\mathrm{C} 14-\mathrm{H} 14 \mathrm{~B}$ & 109.5 \\
\hline $\mathrm{N} 1{ }^{\mathrm{i}}-\mathrm{C} 1-\mathrm{S} 1$ & $122.61(10)$ & $\mathrm{C} 13-\mathrm{C} 14-\mathrm{H} 14 \mathrm{~B}$ & 109.5 \\
\hline $\mathrm{N} 1-\mathrm{C} 1-\mathrm{S} 1$ & $122.61(10)$ & $\mathrm{H} 14 \mathrm{~A}-\mathrm{C} 14-\mathrm{H} 14 \mathrm{~B}$ & 108.1 \\
\hline $\mathrm{N} 1-\mathrm{C} 11-\mathrm{H} 11 \mathrm{~A}$ & 109.5 & $\mathrm{C} 16-\mathrm{C} 15-\mathrm{C} 14$ & $110.50(14)$ \\
\hline $\mathrm{N} 1-\mathrm{C} 11-\mathrm{H} 11 \mathrm{~B}$ & 109.5 & $\mathrm{C} 16-\mathrm{C} 15-\mathrm{H} 15 \mathrm{~A}$ & 109.5 \\
\hline $\mathrm{H} 11 \mathrm{~A}-\mathrm{C} 11-\mathrm{H} 11 \mathrm{~B}$ & 109.5 & $\mathrm{C} 14-\mathrm{C} 15-\mathrm{H} 15 \mathrm{~A}$ & 109.5 \\
\hline $\mathrm{N} 1-\mathrm{C} 11-\mathrm{H} 11 \mathrm{C}$ & 109.5 & $\mathrm{C} 16-\mathrm{C} 15-\mathrm{H} 15 \mathrm{~B}$ & 109.5 \\
\hline $\mathrm{H} 11 \mathrm{~A}-\mathrm{C} 11-\mathrm{H} 11 \mathrm{C}$ & 109.5 & $\mathrm{C} 14-\mathrm{C} 15-\mathrm{H} 15 \mathrm{~B}$ & 109.5 \\
\hline $\mathrm{H} 11 \mathrm{~B}-\mathrm{C} 11-\mathrm{H} 11 \mathrm{C}$ & 109.5 & $\mathrm{H} 15 \mathrm{~A}-\mathrm{C} 15-\mathrm{H} 15 \mathrm{~B}$ & 108.1 \\
\hline $\mathrm{N} 2-\mathrm{C} 2-\mathrm{N} 2^{\mathrm{i}}$ & $114.94(19)$ & $\mathrm{N} 11-\mathrm{C} 16-\mathrm{C} 15$ & $110.17(13)$ \\
\hline $\mathrm{N} 2-\mathrm{C} 2-\mathrm{S} 2$ & $122.53(9)$ & $\mathrm{N} 11-\mathrm{C} 16-\mathrm{H} 16 \mathrm{~A}$ & 109.6 \\
\hline $\mathrm{N} 2 \mathrm{i}-\mathrm{C} 2-\mathrm{S} 2$ & $122.53(9)$ & $\mathrm{C} 15-\mathrm{C} 16-\mathrm{H} 16 \mathrm{~A}$ & 109.6 \\
\hline $\mathrm{C} 12-\mathrm{N} 11-\mathrm{C} 16$ & $112.53(13)$ & $\mathrm{N} 11-\mathrm{C} 16-\mathrm{H} 16 \mathrm{~B}$ & 109.6 \\
\hline $\mathrm{C} 12-\mathrm{N} 11-\mathrm{H} 01$ & $106.8(14)$ & $\mathrm{C} 15-\mathrm{C} 16-\mathrm{H} 16 \mathrm{~B}$ & 109.6 \\
\hline C16-N11-H01 & $111.5(13)$ & $\mathrm{H} 16 \mathrm{~A}-\mathrm{C} 16-\mathrm{H} 16 \mathrm{~B}$ & 108.1 \\
\hline $\mathrm{C} 12-\mathrm{N} 11-\mathrm{H} 02$ & $110.0(14)$ & $\mathrm{O} 2-\mathrm{S} 3-\mathrm{O} 2^{\mathrm{i}}$ & $109.39(9)$ \\
\hline $\mathrm{C} 16-\mathrm{N} 11-\mathrm{H} 02$ & $107.4(14)$ & $\mathrm{O} 2-\mathrm{S} 3-\mathrm{O} 1^{\mathrm{i}}$ & $110.26(6)$ \\
\hline $\mathrm{H} 01-\mathrm{N} 11-\mathrm{H} 02$ & $108.6(19)$ & $\mathrm{O} 2^{\mathrm{i}}-\mathrm{S} 3-\mathrm{O} 1^{\mathrm{i}}$ & $108.74(6)$ \\
\hline $\mathrm{N} 11-\mathrm{C} 12-\mathrm{C} 13$ & $109.67(14)$ & $\mathrm{O} 2-\mathrm{S} 3-\mathrm{O} 1$ & $108.74(6)$ \\
\hline $\mathrm{N} 11-\mathrm{C} 12-\mathrm{H} 12 \mathrm{~A}$ & 109.7 & $\mathrm{O} 2 \mathrm{i}-\mathrm{S} 3-\mathrm{O} 1$ & $110.26(6)$ \\
\hline $\mathrm{C} 13-\mathrm{C} 12-\mathrm{H} 12 \mathrm{~A}$ & 109.7 & $\mathrm{O} 1-\mathrm{S} 3-\mathrm{O} 1$ & $109.46(9)$ \\
\hline $\mathrm{N} 11-\mathrm{C} 12-\mathrm{H} 12 \mathrm{~B}$ & 109.7 & $\mathrm{C} 2-\mathrm{N} 2-\mathrm{C} 21$ & $124.43(14)$ \\
\hline $\mathrm{C} 13-\mathrm{C} 12-\mathrm{H} 12 \mathrm{~B}$ & 109.7 & $\mathrm{C} 2-\mathrm{N} 2-\mathrm{H} 04$ & $118.6(15)$ \\
\hline $\mathrm{H} 12 \mathrm{~A}-\mathrm{C} 12-\mathrm{H} 12 \mathrm{~B}$ & 108.2 & $\mathrm{C} 21-\mathrm{N} 2-\mathrm{H} 04$ & $116.9(15)$ \\
\hline $\mathrm{C} 12-\mathrm{C} 13-\mathrm{C} 14$ & $110.89(15)$ & $\mathrm{N} 2-\mathrm{C} 21-\mathrm{H} 21 \mathrm{~A}$ & 109.5 \\
\hline $\mathrm{C} 12-\mathrm{C} 13-\mathrm{H} 13 \mathrm{~A}$ & 109.5 & $\mathrm{~N} 2-\mathrm{C} 21-\mathrm{H} 21 \mathrm{~B}$ & 109.5 \\
\hline $\mathrm{C} 14-\mathrm{C} 13-\mathrm{H} 13 \mathrm{~A}$ & 109.5 & $\mathrm{H} 21 \mathrm{~A}-\mathrm{C} 21-\mathrm{H} 21 \mathrm{~B}$ & 109.5 \\
\hline $\mathrm{C} 12-\mathrm{C} 13-\mathrm{H} 13 \mathrm{~B}$ & 109.5 & $\mathrm{~N} 2-\mathrm{C} 21-\mathrm{H} 21 \mathrm{C}$ & 109.5 \\
\hline $\mathrm{C} 14-\mathrm{C} 13-\mathrm{H} 13 \mathrm{~B}$ & 109.5 & $\mathrm{H} 21 \mathrm{~A}-\mathrm{C} 21-\mathrm{H} 21 \mathrm{C}$ & 109.5 \\
\hline $\mathrm{H} 13 \mathrm{~A}-\mathrm{C} 13-\mathrm{H} 13 \mathrm{~B}$ & 108.0 & $\mathrm{H} 21 \mathrm{~B}-\mathrm{C} 21-\mathrm{H} 21 \mathrm{C}$ & 109.5 \\
\hline $\mathrm{C} 11-\mathrm{N} 1-\mathrm{C} 1-\mathrm{N} 1^{\mathrm{i}}$ & $-179.1(2)$ & $\mathrm{C} 13-\mathrm{C} 14-\mathrm{C} 15-\mathrm{C} 16$ & $-55.77(19)$ \\
\hline $\mathrm{C} 11-\mathrm{N} 1-\mathrm{C} 1-\mathrm{S} 1$ & $0.9(2)$ & $\mathrm{C} 12-\mathrm{N} 11-\mathrm{C} 16-\mathrm{C} 15$ & $-58.13(18)$ \\
\hline $\mathrm{C} 16-\mathrm{N} 11-\mathrm{C} 12-\mathrm{C} 13$ & $57.99(18)$ & $\mathrm{C} 14-\mathrm{C} 15-\mathrm{C} 16-\mathrm{N} 11$ & $56.16(18)$ \\
\hline $\mathrm{N} 11-\mathrm{C} 12-\mathrm{C} 13-\mathrm{C} 14$ & $-56.3(2)$ & $\mathrm{N} 2 \mathrm{i}-\mathrm{C} 2-\mathrm{N} 2-\mathrm{C} 21$ & $177.95(17)$ \\
\hline $\mathrm{C} 12-\mathrm{C} 13-\mathrm{C} 14-\mathrm{C} 15$ & $56.1(2)$ & $\mathrm{S} 2-\mathrm{C} 2-\mathrm{N} 2-\mathrm{C} 21$ & $-2.05(17)$ \\
\hline
\end{tabular}

Symmetry code: (i) $-x+1, y,-z+1 / 2$.

Hydrogen-bond geometry $\left(A,{ }^{\circ}\right)$

\begin{tabular}{lllll}
\hline$D-\mathrm{H} \cdots A$ & $D-\mathrm{H}$ & $\mathrm{H} \cdots A$ & $D \cdots A$ & $D-\mathrm{H} \cdots A$ \\
\hline $\mathrm{N} 1-\mathrm{H} 03 \cdots \mathrm{O} 1$ & $0.86(3)$ & $2.06(3)$ & $2.9062(18)$ & $173(2)$ \\
$\mathrm{N} 2-\mathrm{H} 04 \cdots \mathrm{O} 2$ & $0.89(2)$ & $2.00(2)$ & $2.8874(17)$ & $172(2)$
\end{tabular}


supporting information

\begin{tabular}{lllll}
$\mathrm{N} 11-\mathrm{H} 01 \cdots \mathrm{O} 1^{\mathrm{ii}}$ & $0.83(2)$ & $1.98(2)$ & $2.7953(18)$ & $167.4(19)$ \\
$\mathrm{N} 11-\mathrm{H} 02 \cdots \mathrm{O} 2$ & $0.91(2)$ & $1.85(2)$ & $2.7589(17)$ & $176(2)$ \\
$\mathrm{C} 12-\mathrm{H} 12 A \cdots \mathrm{S} 1^{\mathrm{iii}}$ & 0.99 & 2.94 & $3.8213(17)$ & 150 \\
$\mathrm{C} 15-\mathrm{H} 15 A \cdots \mathrm{O} 2^{\mathrm{iv}}$ & 0.99 & 2.49 & $3.435(2)$ & 158 \\
\hline
\end{tabular}

Symmetry codes: (ii) $-x+1,-y+1,-z+1$; (iii) $x-1 / 2, y+1 / 2, z$; (iv) $x,-y+1, z+1 / 2$. 